\title{
Correction to: Advances in Rasch Analyses in the Human Sciences
}

\author{
Correction to: \\ W. J. Boone, J. R. Staver, Advances in Rasch \\ Analyses in the Human Sciences, \\ https://doi.org/10.1007/978-3-030-43420-5
}

An earlier version of the book was inadvertently published in which the availability of the extra supplementary material was incorrectly mentioned in the following pages of the book:

x, xi, xii, xiii, xiv, xv, xvi, 22, 36, 53, 62, 77, 92, 108, 126, 143, 157, 170, 212, 249, 298.

It has now been corrected and the links has been replaced as follows:

Electronic Supplementary Material: The online version of this chapter (https:// doi.org/10.1007/978-3-030-43420-5_2) contains supplementary material, which is available to authorized users.

Electronic Supplementary Material: The online version of this chapter (https:// doi.org/10.1007/978-3-030-43420-5_3) contains supplementary material, which is available to authorized users.

Electronic Supplementary Material: The online version of this chapter (https:// doi.org/10.1007/978-3-030-43420-5_4) contains supplementary material, which is available to authorized users.

Electronic Supplementary Material: The online version of this chapter (https:// doi.org/10.1007/978-3-030-43420-5_5) contains supplementary material, which is available to authorized users.

The updated version of the book can found at

https://doi.org/10.1007/978-3-030-43420-5 
Electronic Supplementary Material: The online version of this chapter (https:// doi.org/10.1007/978-3-030-43420-5_6) contains supplementary material, which is available to authorized users.

Electronic Supplementary Material: The online version of this chapter (https:// doi.org/10.1007/978-3-030-43420-5_7) contains supplementary material, which is available to authorized users.

Electronic Supplementary Material: The online version of this chapter (https:// doi.org/10.1007/978-3-030-43420-5_8) contains supplementary material, which is available to authorized users.

Electronic Supplementary Material: The online version of this chapter (https:// doi.org/10.1007/978-3-030-43420-5_9) contains supplementary material, which is available to authorized users.

Electronic Supplementary Material: The online version of this chapter (https:// doi.org/10.1007/978-3-030-43420-5_10) contains supplementary material, which is available to authorized users.

Electronic Supplementary Material: The online version of this chapter (https:// doi.org/10.1007/978-3-030-43420-5_11) contains supplementary material, which is available to authorized users.

Electronic Supplementary Material: The online version of this chapter (https:// doi.org/10.1007/978-3-030-43420-5_15) contains supplementary material, which is available to authorized users.

Electronic Supplementary Material: The online version of this chapter (https:// doi.org/10.1007/978-3-030-43420-5_16) contains supplementary material, which is available to authorized users.

Electronic Supplementary Material: The online version of this chapter (https:// doi.org/10.1007/978-3-030-43420-5_19) contains supplementary material, which is available to authorized users. 